\title{
LIMIT THEOREMS FOR A GENERALIZED FELLER GAME
}

\author{
KEISUKE MATSUMOTO $* * *$ AND \\ TOSHIO NAKATA, ${ }^{* * *}$ Fukuoka University of Education
}

\begin{abstract}
In this paper we study limit theorems for the Feller game which is constructed from onedimensional simple symmetric random walks, and corresponds to the St. Petersburg game. Motivated by a generalization of the St. Petersburg game which was investigated by Gut (2010), we generalize the Feller game by introducing the parameter $\alpha$. We investigate limit distributions of the generalized Feller game corresponding to the results of Gut. Firstly, we give the weak law of large numbers for $\alpha=1$. Moreover, for $0<\alpha \leq 1$, we have convergence in distribution to a stable law with index $\alpha$. Finally, some limit theorems for a polynomial size and a geometric size deviation are given.
\end{abstract}

Keywords: Feller's game; simple symmetric random walk; law of large numbers; stable law; extremes

2010 Mathematics Subject Classification: Primary 60F05

Secondary 60G50

\section{Introduction}

\subsection{The Feller game and the St. Petersburg game}

Feller wrote the following coin tossing procedure in his textbook [5, Section X.2, p. 231].

For $n \in \mathbb{N}:=\{1,2,3, \ldots\}$, suppose that $n$ coins are tossed one by one. For $i=1,2, \ldots, n$, consider the waiting time for the $i$ th coin up to the first equalization of the accumulated numbers of heads and tails.

We assume that if the waiting time is $i=2 k$ then the player receives $2 k$ yen for $k \in \mathbb{N}$. Then, the coin tossing game is regarded as a game of chance which corresponds to the St. Petersburg game. So, we will call it the Feller game. Indeed, in the St. Petersburg game a fair coin is tossed repeatedly until the first head appears. If this happens at the $k$ th trial then the player receives $2^{k}$ yen (see [5, Section X.4, p. 235]).

By the definition of the Feller game, it is formulated as the sum of independent and identically distributed (i.i.d.) random variables which are distributed according to the law of the return times of one-dimensional simple symmetric random walks from the origin. Since the setting is natural, the Feller game is described in some standard textbooks of probability theory (see [15, Chapter 4, Theorem 4.8.3], [2, Section 9.12, Theorem 9.40], [4, Example 3.7.2], and [17, Example 1.0.1, p. 25]). For the St. Petersburg game, a natural fair price to participate would be the expected value, which is infinite, as in the Feller game. Let $\left\{X_{i}\right\}$ be i.i.d. random variables

Received 23 February 2012; revision received 26 June 2012.

* Postal address: Department of Mathematics, Fukuoka University of Education, Akama-Bunkyomachi, Munakata,

Fukuoka, 811-4192, Japan.

** Email address: mk234704@goo.jp

*** Email address: nakata@ fukuoka-edu.ac.jp 
which denote the waiting times of the $i$ th coin in the Feller game. The common distribution of $\left\{X_{i}\right\}$ is

$$
\mathbb{P}(X=2 k)=\frac{1}{2 k-1}\left(\begin{array}{c}
2 k \\
k
\end{array}\right) 2^{-2 k} \text { for } k=1,2, \ldots
$$

from a general theory of random walks (see, e.g. [6, Exercises 3.10.1, p. 83]). Similarly, let $\left\{Y_{i}\right\}$ be i.i.d. random variables which denote the waiting times in the St. Petersburg game. By definition, the common distribution of $\left\{Y_{i}\right\}$ is

$$
\mathbb{P}\left(Y=2^{k}\right)=2^{-k} \text { for } k=1,2, \ldots
$$

For (1) and (2), we have $\mathbb{E} X=\mathbb{E} Y=\infty$, so these two games have a similar property in that they are of infinite expectation. The fact that $\mathbb{E} Y=\infty$ is trivial. The fact that $\mathbb{E} X=\infty$ follows from the null recurrence property of simple symmetric random walks (see [14, Example 1.7.8]). The direct proof of $\mathbb{E} X=\infty$ will be given in Lemma 2 .

The St. Petersburg and Feller games have different properties. One difference between the two games concerns the regular variation at $\infty$ of the tail probability. For the St. Petersburg game, the tail probability $\mathbb{P}(Y>y)$ for $y>1$ of (2) satisfies

$$
\mathbb{P}(Y>y)=\sum_{2^{k}>y} 2^{-k}=\sum_{k=\left\lfloor\log _{2} y\right\rfloor+1}^{\infty} 2^{-k}=2^{-\left\lfloor\log _{2} y\right\rfloor}=y^{-1} 2^{\left\{\log _{2} y\right\}},
$$

where, for $x \in \mathbb{R}^{+}:=(0,+\infty)$, the notation $\lfloor x\rfloor$ denotes the integer part of $x$, and the notation $\{x\}$ denotes the fractional part of $x$. Note that $1 \leq 2^{\left\{\log _{2} y\right\}}<2$, which is the coefficient of $y^{-1}$ in (3), fluctuates depending on $y$. Thus, (3) is not regularly varying at $\infty$ (see Definition 1), a fact that was also noted in [3, p. 373]. So, (3) is intractable because we cannot apply standard convergence theorems concerning both stable distributions and extreme distributions (see, e.g. [4, Theorem 3.7.2] and [8, Theorem 9.6.3 (a)]). Therefore, the proof of Theorem 2.1(ii) and (iii) of [9] should be reexamined. For the Feller game, the tail probability $\mathbb{P}(X>x)$ for $x \in \mathbb{R}^{+}$ of (1) is regularly varying at $\infty$ (see Lemma 2 ). Hence, we can easily apply standard theorems to the Feller game.

\subsection{Known results}

1.2.1. The law of large numbers. To the authors' knowledge, the law of large numbers has not been established for the Feller game. On the other hand, the weak law of large numbers was established for the St. Petersburg game by Feller [5, Section X.4, Equation (4.1)]. Let $T_{n}:=\sum_{i=1}^{n} Y_{i}$ be the total gain for $n$ coins, where $\left\{Y_{i}\right\}$ is a sequence of i.i.d. random variables with common distribution (2). Feller showed that

$$
\frac{T_{n}}{n \log _{2} n} \stackrel{\mathbb{P}}{\rightarrow} 1 \quad \text { as } n \rightarrow \infty
$$

where $\stackrel{\mathbb{P}}{\rightarrow}$ ' denotes convergence in probability. In other words, $T_{n}$ is relatively stable with the normalizing constant $n \log _{2} n$ (see [3, Section 8.8] and [11]). Moreover, Adler [1, Example 4] showed the following results concerning almost-sure convergence:

$$
\liminf _{n \rightarrow \infty} \frac{T_{n}}{n \log _{2} n}=1 \quad \text { almost surely, } \quad \limsup _{n \rightarrow \infty} \frac{T_{n}}{n \log _{2} n}=\infty \quad \text { almost surely. }
$$


1.2.2. The limit distributions. For the Feller game, let $\left\{X_{i}\right\}$ be a sequence of i.i.d. random variables with distribution (1), and let $S_{n}:=\sum_{i=1}^{n} X_{i}$. Then, the following limit distribution is known.

Theorem 1. ([5, Theorem 2, Equation (6.8), p. 83].) We have

$$
\lim _{n \rightarrow \infty} \mathbb{P}\left(\frac{S_{n}}{n^{2}}<x\right)=\sqrt{\frac{2}{\pi}} \int_{1 / \sqrt{x}}^{\infty} \mathrm{e}^{-s^{2} / 2} \mathrm{~d} s=2\left\{1-\mathcal{N}\left(\frac{1}{\sqrt{x}}\right)\right\} \text { for } x>0,
$$

where $\mathcal{N}(\alpha):=\int_{-\infty}^{\alpha} \mathrm{e}^{-x^{2} / 2} / \sqrt{2 \pi} \mathrm{d} x$.

A direct proof of Theorem 1 was given in [5, Theorem 2, Equation (6.8), p. 83]). See also [2, Section 9.12, Theorem 9.40], [4, Example 3.7.2], [15, Chapter 4, Theorem 4.8.3], and [17, Example 1.0.1]. Moreover, the density function of the limit distribution (6) is $\mathrm{e}^{-1 /(2 x)} /\left(\sqrt{2 \pi} x^{3 / 2}\right)$ for $x>0$, which is the one-sided stable Lévy distribution of index $\frac{1}{2}$ (see $[13$, p. 5$]$ ).

For the St. Petersburg game, a limit distribution along the subsequence $2^{n}$ was given by Martin-Löf [12].

Theorem 2. ([12, Theorem 1].) For the random variable $T_{n}$ in the St. Petersburg game and $N:=2^{n}$, we have

$$
\frac{T_{N}-N \log _{2} N}{N}=\frac{T_{N}}{N}-n \stackrel{\mathrm{D}}{\rightarrow} Z \quad \text { as } n \rightarrow \infty,
$$

where $\stackrel{\mathrm{D}}{\rightarrow}$ ' denotes convergence in distribution and $\mathrm{Z}$ is defined via the characteristic function

$$
\log \mathbb{E}\left(\mathrm{e}^{\mathrm{i} u Z}\right)=\sum_{k=-\infty}^{0}\left(\exp \left\{\mathrm{i} u 2^{k}\right\}-1-\mathrm{i} u 2^{k}\right) 2^{-k}+\sum_{k=1}^{\infty}\left(\exp \left\{\mathrm{i} u 2^{k}\right\}-1\right) 2^{-k}
$$

Note that $\log (\cdot)$ denotes the natural logarithm throughout the paper. The distribution of $Z$ is infinitely divisible. Moreover, the Lévy measure has $2^{-k}$ atoms at the points $2^{k}$ for $k=0, \pm 1, \pm 2, \ldots$. This distribution is not stable but semistable in the sense of Paul Lévy (see [12, Theorem 2]).

\subsection{A generalization of the Feller game}

Motivated by [9], in this paper we consider a generalization of the probability distribution (1) by introducing the parameter $\alpha>0$ :

$$
\mathbb{P}\left(X^{(\alpha)}=(2 k)^{1 /(2 \alpha)}\right)=\frac{1}{2 k-1}\left(\begin{array}{c}
2 k \\
k
\end{array}\right) 2^{-2 k} \text { for } k=1,2, \ldots
$$

Actually, the amount concerning the Feller game is adjusted by $\alpha$. We call it a generalized Feller game. Note that if $\alpha=\frac{1}{2}$, the probability distribution (7) is equivalent to (1). Let $\left\{X_{i}^{(\alpha)}\right\}$ be a sequence of i.i.d. random variables with distribution (7). Define the random variables

$$
S_{n}^{(\alpha)}:=\sum_{k=1}^{n} X_{k}^{(\alpha)} \text { and } \quad M_{n}^{(\alpha)}:=\max _{1 \leq k \leq n} X_{k}^{(\alpha)},
$$

which respectively denote the total gain for $n$ coins and the maximal gain for $n$ coins. We will study some statistical properties of both $S_{n}^{(\alpha)}$ and $M_{n}^{(\alpha)}$. 
We focus on the case $0<\alpha \leq 1$. If $\alpha>1$ then $\mathbb{E} X^{(\alpha)}<+\infty$ holds (see (19) below). Then we can obtain the strong law of large numbers, but the essential property of the Feller game, $\mathbb{E} X^{(\alpha)}=+\infty$, is lost.

The main goal of this paper is to give the corresponding results of [9, Theorems 2.1, 6.1, and 6.2] with respect to the generalized Feller game for $0<\alpha \leq 1$. In Theorem 3(i), $S_{n}^{(1)}$ is relatively stable, as well as $T_{n}$ in (4), namely, $S_{n}^{(1)} /(\sqrt{2 / \pi} n \log n) \stackrel{\mathbb{P}}{\rightarrow} 1$ as $n \rightarrow \infty$ via the Gut-Kolmogorov-Feller theorem (see [7, Theorem 1.3] or [8, Theorem 6.4.2]). However, if $0<\alpha<1$ then $S_{n}^{(\alpha)}$ is not relatively stable. Namely, the weak law of large numbers with any suitable constant does not hold, which is easily proved using [11, Theorem 1]. In Proposition 1 we show that the strong law of large numbers with respect to $S_{n}^{(1)}$ does not hold. It corresponds to (5). In Theorem 3(ii) we show that an appropriately normalized $S_{n}^{(\alpha)}$ converges in distribution to a stable law with index $\alpha$ for $0<\alpha \leq 1$. Comparing Theorem 2 and Theorem 3(ii) for $\alpha=1$, we confirm that the Feller game and the St. Petersburg game have different properties. In Theorem 3(iii) we show that $M_{n}^{(\alpha)}$ converges in distribution to the Fréchet distribution (see, e.g. [8, p. 452]) for $0<\alpha \leq 1$. Finally, referring to results in [10] and [16], we give results for a polynomial size and a geometric size deviation of $S_{n}^{(\alpha)}$ and $M_{n}^{(\alpha)}$ in Section 5. The arguments are the same as those in [9, Theorems 6.1 and 6.2].

\section{Main results}

To state our main theorem, we need the sign function

$$
\operatorname{sgn}(t):= \begin{cases}-1, & t<0 \\ 0, & t=0 \\ 1, & t>0\end{cases}
$$

Theorem 3. (i) The weak law of large numbers:

- if $0<\alpha<1$ then $S_{n}^{(\alpha)}$ is not relatively stable, namely, there are no sequences $\left\{a_{n}\right\}$ that satisfy

$$
\frac{S_{n}^{(\alpha)}}{a_{n}} \stackrel{\mathbb{P}}{\rightarrow} 1 \text { as } n \rightarrow \infty,
$$

- if $\alpha=1$ then $S_{n}^{(1)}$ is relatively stable with normalizing constant $\sqrt{2 / \pi} n \log n$, namely, we have

$$
\frac{S_{n}^{(1)}}{\sqrt{2 / \pi} n \log n} \stackrel{\mathbb{P}}{\rightarrow} 1 \text { as } n \rightarrow \infty .
$$

(ii) The stable laws:

- if $0<\alpha<1$ then we have

$$
\left(\frac{\pi}{2}\right)^{1 /(2 \alpha)} \frac{S_{n}^{(\alpha)}}{n^{1 / \alpha}} \stackrel{\mathrm{D}}{\rightarrow} Z^{(\alpha)} \quad \text { as } n \rightarrow \infty,
$$

where $Z^{(\alpha)}$ is the random variable whose distribution is stable with index $\alpha$ defined via the characteristic function

$$
\mathbb{E}\left(\mathrm{e}^{\mathrm{i} t Z^{(\alpha)}}\right)=\exp \left\{\mathrm{i} t c-b|t|^{\alpha}\left(1+\mathrm{i} \operatorname{sgn}(t) \tan \left(\frac{\pi \alpha}{2}\right)\right)\right\}
$$

for some $c \in \mathbb{R}$ and $b>0$, 
- if $\alpha=1$ then we have

$$
\sqrt{\frac{\pi}{2}} \frac{S_{n}^{(1)}}{n}-\log n \stackrel{\mathrm{D}}{\rightarrow} Z^{(1)} \quad \text { as } n \rightarrow \infty,
$$

where $Z^{(1)}$ is the random variable whose distribution is stable with index 1 defined via the characteristic function

$$
\mathbb{E}\left(\mathrm{e}^{\mathrm{i} t Z^{(1)}}\right)=\exp \left(\mathrm{i} t c^{\prime}-b^{\prime}|t|\left\{1+\mathrm{i} \operatorname{sgn}(t)\left(\frac{2}{\pi}\right) \log |t|\right\}\right)
$$

for some $c^{\prime} \in \mathbb{R}$ and $b^{\prime}>0$.

(iii) The extreme laws: if $0<\alpha \leq 1$ then we have

$$
\left(\frac{\pi}{2}\right)^{1 /(2 \alpha)} \frac{M_{n}^{(\alpha)}}{n^{1 / \alpha}} \stackrel{\mathrm{D}}{\rightarrow} \Phi_{\alpha} \quad \text { as } n \rightarrow \infty,
$$

where $\Phi_{\alpha}(x)=\exp \left\{-x^{-\alpha}\right\}$ for $x>0$ is the Fréchet distribution.

Note that $0 \log 0$ is interpreted as 0 in (13). The following result is analogous to (5).

Proposition 1. We have

$$
\begin{aligned}
& \limsup _{n \rightarrow \infty} \frac{S_{n}^{(1)}}{\sqrt{2 / \pi} n \log n}=\infty \quad \text { almost surely, } \\
& \liminf _{n \rightarrow \infty} \frac{S_{n}^{(1)}}{\sqrt{2 / \pi} n \log n}=1 \quad \text { almost surely. }
\end{aligned}
$$

\section{Preliminaries}

For $0<\alpha \leq 1$, we investigate the tail probability of $X^{(\alpha)}$. By the Stirling formula (see [5, Equation (9.8)]) we have

$$
\mathbb{P}\left(X^{(\alpha)}=2 k\right)=\frac{1}{2 \sqrt{\pi}} k^{-3 / 2}\left(1+O\left(k^{-1}\right)\right),
$$

where $a_{k}=O\left(b_{k}\right)$ denotes $\lim \sup _{k \rightarrow \infty} a_{k} / b_{k}<\infty$.

To approximate the sums of positive terms by integration, we state without proof the following elementary lemma.

Lemma 1. Let $f: \mathbb{R}^{+} \rightarrow \mathbb{R}^{+}$be a continuous function. Then the following statements hold.

(i) If $f$ is monotone increasing or monotone decreasing then, for $s>1$,

$$
\left|\sum_{1 \leq k \leq\lfloor s\rfloor} f(k)-\int_{1}^{\lfloor s\rfloor+1} f(t) \mathrm{d} t\right| \leq|f(1)-f(\lfloor s\rfloor+1)| .
$$

(ii) If $f$ is monotone decreasing and $\sum_{k=1}^{\infty} f(k)<+\infty$, then, for $s>0$,

$$
\int_{s+1}^{\infty} f(t) \mathrm{d} t \leq \sum_{k>s} f(k) \leq \int_{s}^{\infty} f(t) \mathrm{d} t+f(s) .
$$


Using Lemma 1 and (16), we examine the statistical properties of $X^{(\alpha)}$.

Lemma 2. For $X^{(\alpha)}$ defined by (7), we have

$$
\mathbb{E} X^{(\alpha)}\left\{\begin{array} { l } 
{ < \infty \text { for } \alpha > 1 , } \\
{ = \infty \text { for } 0 < \alpha \leq 1 , }
\end{array} \mathbb { E } ( ( X ^ { ( \alpha ) } ) ^ { \beta } ) \left\{\begin{array}{ll}
<\infty & \text { for } 0<\beta<\alpha \leq 1, \\
=\infty & \text { for } \beta \geq \alpha
\end{array}\right.\right.
$$

Moreover, as $x \rightarrow \infty$, we have

$$
\begin{aligned}
& \mathbb{P}\left(X^{(\alpha)}>x\right) \sim \sqrt{\frac{2}{\pi}} x^{-\alpha} \text { for } \alpha>0, \\
& \mathbb{E} X^{(\alpha)} \mathbf{1}\left\{X^{(\alpha)} \leq x\right\} \sim \begin{cases}\sqrt{\frac{2}{\pi}} \log x & \text { for } \alpha=1, \\
\frac{\alpha}{1-\alpha} \sqrt{\frac{2}{\pi}} x^{1-\alpha} & \text { for } 0<\alpha<1,\end{cases} \\
& \mathbb{E}\left(X^{(\alpha)}\right)^{2} \mathbf{1}\left\{X^{(\alpha)} \leq x\right\} \sim \frac{\alpha}{2-\alpha} \sqrt{\frac{2}{\pi}} x^{2-\alpha} \quad \text { for } 0<\alpha \leq 1,
\end{aligned}
$$

where $f(x) \sim g(x)$ denotes

$$
\lim _{x \rightarrow \infty} \frac{f(x)}{g(x)}=1 .
$$

Proof. For $\alpha>0$ and $\beta>0$, (16) yields

$$
\mathbb{E}\left(\left(X^{(\alpha)}\right)^{\beta}\right)=\sum_{k=1}^{\infty}(2 k)^{\beta /(2 \alpha)} \frac{1}{2 \sqrt{\pi}} k^{-3 / 2}\left(1+O\left(k^{-1}\right)\right)=C \sum_{k=1}^{\infty} k^{\beta /(2 \alpha)-3 / 2}\left(1+O\left(k^{-1}\right)\right),
$$

where $C>0$ is some constant. Since

$$
\sum_{k=1}^{\infty} k^{\gamma} \begin{cases}<\infty & \text { for } \gamma<-1 \\ =\infty & \text { for } \gamma \geq-1\end{cases}
$$

we have (19). Next, for sufficiently large $x \in \mathbb{R}^{+}$, using (16), we see that

$$
\mathbb{P}\left(X^{(\alpha)}>x\right)=\sum_{k>x^{2 \alpha} / 2} \frac{1}{2 \sqrt{\pi}} k^{-3 / 2}\left(1+O\left(k^{-1}\right)\right) \sim \sqrt{\frac{2}{\pi}} x^{-\alpha} .
$$

We check the ' $\sim$ ' of (23). Equation (18) implies that

$$
\begin{aligned}
2 \sqrt{2} x^{-\alpha}\left(1+\frac{2}{x^{2 \alpha}}\right)^{-1 / 2} & =\int_{x^{2 \alpha} / 2+1}^{\infty} t^{-3 / 2} \mathrm{~d} t \\
& \leq \sum_{k>x^{2 \alpha} / 2} k^{-3 / 2} \\
& \leq \int_{x^{2 \alpha} / 2}^{\infty} t^{-3 / 2} \mathrm{~d} t+\left(\frac{x^{2 \alpha}}{2}\right)^{-3 / 2} \\
& =2 \sqrt{2}\left(x^{-\alpha}+x^{-3 \alpha}\right) .
\end{aligned}
$$


By the definition of ' $\sim$ ' (see (22)), we have $\sum_{k>x^{2 \alpha} / 2} k^{-3 / 2} \sim 2 \sqrt{2} x^{-\alpha}$. This implies (23). Moreover, for sufficiently large $x \in \mathbb{R}^{+}$, using (16), we also obtain

$$
\mathbb{E} X^{(\alpha)} \mathbf{1}\left\{X^{(\alpha)} \leq x\right\}=\frac{2^{1 /(2 \alpha)-1}}{\sqrt{\pi}} \sum_{k=1}^{\left\lfloor x^{2 \alpha} / 2\right\rfloor} k^{1 /(2 \alpha)-3 / 2}\left(1+O\left(k^{-1}\right)\right) .
$$

If $\alpha=1$ then we have

$$
\mathbb{E} X^{(1)} \mathbf{1}\left\{X^{(1)} \leq x\right\}=\frac{1}{\sqrt{2 \pi}} \int_{1}^{\left\lfloor x^{2} / 2\right\rfloor} \frac{1}{t} \mathrm{~d} t+O(1) \sim \sqrt{\frac{2}{\pi}} \log x .
$$

If $0<\alpha<1$ then we see that

$$
\mathbb{E} X^{(\alpha)} \mathbf{1}\left\{X^{(\alpha)} \leq x\right\} \sim \frac{2^{1 /(2 \alpha)-1}}{\sqrt{\pi}} \int_{1}^{\left\lfloor x^{2 \alpha} / 2\right\rfloor} t^{1 /(2 \alpha)-3 / 2} \mathrm{~d} t \sim \frac{\alpha}{1-\alpha} \sqrt{\frac{2}{\pi}} x^{1-\alpha} .
$$

We check the first ' $\sim$ ' of (24). From (17) we obtain

$$
\left|\sum_{k=1}^{\left\lfloor x^{2 \alpha} / 2\right\rfloor} k^{1 /(2 \alpha)-3 / 2}-\int_{1}^{\left\lfloor x^{2 \alpha} / 2\right\rfloor} t^{1 /(2 \alpha)-3 / 2} \mathrm{~d} t\right| \leq\left|1-\left(\left\lfloor\frac{x^{2 \alpha}}{2}\right\rfloor+1\right)^{(1-3 \alpha) /(2 \alpha)}\right|
$$

The integral part of (25) is

$$
\int_{1}^{\left\lfloor x^{2 \alpha} / 2\right\rfloor} t^{1 /(2 \alpha)-3 / 2} \mathrm{~d} t=\frac{2 \alpha}{1-\alpha}\left\lfloor\frac{x^{2 \alpha}}{2}\right\rfloor^{(1-\alpha) /(2 \alpha)}-\frac{2 \alpha}{1-\alpha} .
$$

Hence, dividing both sides of (25) by (26), and letting $x \rightarrow \infty$, we obtain the first ' $~$ ' of (24). The second ' $\sim$ ' of (24) is also given by (26).

By the same arguments, we have, for all $0<\alpha \leq 1$,

$$
\begin{aligned}
\mathbb{E}\left(X^{(\alpha)}\right)^{2} \mathbf{1}\left\{X^{(\alpha)} \leq x\right\} & =\sum_{k=1}^{\left\lfloor x^{2 \alpha} / 2\right\rfloor}(2 k)^{1 / \alpha} \frac{1}{2 \sqrt{\pi}} k^{-3 / 2}\left(1+O\left(k^{-1}\right)\right) \\
& \sim \frac{2^{1 / \alpha-1}}{\sqrt{\pi}} \int_{1}^{\left\lfloor x^{2 \alpha} / 2\right\rfloor} t^{1 / \alpha-3 / 2} \mathrm{~d} t \\
& \sim \frac{\alpha}{2-\alpha} \sqrt{\frac{2}{\pi}} x^{2-\alpha} .
\end{aligned}
$$

This completes the proof.

We also need the concepts of regularly varying and slowly varying functions.

Definition 1. Let $a^{*} \in \mathbb{R}^{+}$. A positive function $u$ on $\left[a^{*}, \infty\right)$ varies regularly at $\infty$ with exponent $\rho(-\infty<\rho<\infty)$, denoted by $u \in \mathcal{R} \mathcal{V}(\rho)$, if

$$
\lim _{t \rightarrow \infty} \frac{u(t x)}{u(t)}=x^{\rho} \quad \text { for all } x \in \mathbb{R}^{+} .
$$

If $\rho=0$ then the function is slowly varying at $\infty$, denoted by $u \in \delta \mathcal{V}$.

For details of regularly and slowly varying functions, we refer the reader to [3] and [8, Section A.7]. 


\section{Proofs of Theorem 3 and Proposition 1}

Proof of Theorem 3. (i) Suppose that $\alpha=1$. Then, from (20) we see that

$$
\lim _{n \rightarrow \infty} n \mathbb{P}\left(X^{(1)}>n \log n\right)=\lim _{n \rightarrow \infty} \sqrt{\frac{2}{\pi}} n\left(\sqrt{\frac{2}{\pi}} n \log n\right)^{-1}=0 .
$$

In the Gut-Kolmogorov-Feller theorem (see [7, Theorem 1.3]), we choose

$$
b(x)=\sqrt{\frac{2}{\pi}} x \log x .
$$

Indeed, by Definition 1 we have $b(x) \in \mathcal{R} \mathcal{V}(1)$. Therefore, since we can apply the GutKolmogorov-Feller theorem to this case, it follows that

$$
\frac{S_{n}^{(1)}-n \mathbb{E} X^{(1)} \mathbf{1}\left\{X^{(1)} \leq \sqrt{2 / \pi} n \log n\right\}}{\sqrt{2 / \pi} n \log n} \stackrel{\mathbb{P}}{\rightarrow} 0 \quad \text { as } n \rightarrow \infty .
$$

By (21) we have

$$
\lim _{n \rightarrow \infty} \frac{n \mathbb{E} X^{(1)} \mathbf{1}\left\{X^{(1)} \leq \sqrt{2 / \pi} n \log n\right\}}{\sqrt{2 / \pi} n \log n}=1 .
$$

Using this and (27) we obtain (9).

Next, suppose that $0<\alpha<1$. Note that $\mathbb{P}\left(\left|X^{(\alpha)}\right|>x\right)=\mathbb{P}\left(X^{(\alpha)}>x\right)>0$ for every $x>0$. Moreover, by (20) and (21), we have

$$
\lim _{x \rightarrow \infty} \frac{x \mathbb{P}\left(\left|X^{(\alpha)}\right|>x\right)}{\mathbb{E} X^{(\alpha)} \mathbf{1}\left\{\left|X^{(\alpha)}\right| \leq x\right\}}=\lim _{x \rightarrow \infty} \frac{x \mathbb{P}\left(X^{(\alpha)}>x\right)}{\mathbb{E} X^{(\alpha)} \mathbf{1}\left\{X^{(\alpha)} \leq x\right\}}=\frac{1-\alpha}{\alpha} \neq 0 .
$$

Hence, [11, Theorem 1] yields (8).

(ii) For $0<\alpha \leq 1$, we confirm the assumptions of [4, Theorem 3.7.2] with respect to $X^{(\alpha)}$. Namely, we check the following two conditions.

(a) $\theta:=\lim _{x \rightarrow \infty} \mathbb{P}\left(X^{(\alpha)}>x\right) / \mathbb{P}\left(\left|X^{(\alpha)}\right|>x\right)=1 \in[0,1]$,

(b) $\mathbb{P}\left(\left|X^{(\alpha)}\right|>x\right)=\mathbb{P}\left(X^{(\alpha)}>x\right)=x^{-\alpha} L(x)$ for $\alpha<2$ and $L \in \& \mathcal{V}$.

Condition (a) follows from $\mathbb{P}\left(X^{(\alpha)}>0\right)=1$. By (20) and (22), there exists $\delta(x)$ satisfying $\mathbb{P}\left(X^{(\alpha)}>x\right)=\sqrt{2 / \pi} x^{-\alpha}(1+\delta(x))$ and $\lim _{x \rightarrow \infty} \delta(x)=0$. If we put $L(x)=\sqrt{2 / \pi}(1+$ $\delta(x))$ then, owing to Definition $1, L \in \delta \mathcal{V}$. Therefore, condition (b) also follows. Hence, we can apply [4, Theorem 3.7.2] to $X^{(\alpha)}$ for all $0<\alpha \leq 1$. Indeed, for two sequences $\left\{a_{n}\right\}$ and $\left\{b_{n}\right\}$, defined by

$$
a_{n}:=\inf \left\{x: \mathbb{P}\left(\left|X^{(\alpha)}\right|>x\right) \leq n^{-1}\right\}, \quad b_{n}:=n \mathbb{E} X^{(\alpha)} \mathbf{1}\left\{\left|X^{(\alpha)}\right| \leq a_{n}\right\},
$$

we have $\left(S_{n}^{(\alpha)}-b_{n}\right) / a_{n} \stackrel{\mathrm{D}}{\rightarrow} Z^{(\alpha)}$ as $n \rightarrow \infty$, where the distribution of $Z^{(\alpha)}$ is nondegenerate. We explicitly determine $\left\{a_{n}\right\}$ and $\left\{b_{n}\right\}$ for $0<\alpha \leq 1$. By (20) and (28), solving $\sqrt{2 / \pi} x^{-\alpha} \leq$ $1 / n$ we obtain, for $0<\alpha \leq 1$,

$$
a_{n}=\left(\frac{2}{\pi}\right)^{1 /(2 \alpha)} n^{1 / \alpha}=\left(\sqrt{\frac{2}{\pi}} n\right)^{1 / \alpha}
$$


On the other hand, (21) and (28) imply that

$$
b_{n}=n \frac{\alpha}{1-\alpha} \sqrt{\frac{2}{\pi}}\left\{\left(\sqrt{\frac{2}{\pi}} n\right)^{1 / \alpha}\right\}^{1-\alpha}=\frac{\alpha}{1-\alpha}\left(\sqrt{\frac{2}{\pi}} n\right)^{1 / \alpha} \quad \text { for } 0<\alpha<1 .
$$

Hence, we obtain (10). If $\alpha=1$ then we have $b_{n}=n \sqrt{2 / \pi} \log (\sqrt{2 / \pi} n)$ by (21) and (28). This implies (12).

The characteristic function of $Z^{(\alpha)}$ is given by [4, Equation (3.7.11)] with $\kappa=2 \theta-1=1$. Hence, if $0<\alpha<1$, we obtain (11) and if $\alpha=1$, we obtain (13).

(iii) For $0<\alpha \leq 1$, (20) implies $\mathbb{P}\left(X^{(\alpha)}>x\right) \in \mathcal{R V}(-\alpha)$. Hence, applying [8, Theorem 9.6.3 (a)] yields (14).

Proof of Proposition 1. To show (15), we quote [1, Theorem 2 and Example 4]. In the notation of [1, Theorem 2], $\alpha=0, \mu(x) \sim \log x$, and $c_{n}=(1+o(1)) \sqrt{2 / \pi} n \log n$, where $e_{n}=o\left(d_{n}\right)$ denotes $\lim _{n \rightarrow \infty} e_{n} / d_{n}=0$. Since all the hypotheses of [1, Theorem 2] hold, we have (15).

\section{Polynomial size and geometric size deviations}

For a polynomial size deviation, we have the following result.

Theorem 4. ([9, Theorem 6.1].) For $0<\alpha \leq 1$, consider $b \in \mathbb{R}^{+}$satisfying $b>1 / \alpha$. Then we have

$$
\lim _{n \rightarrow \infty} \frac{\log \mathbb{P}\left(S_{n}^{(\alpha)}>n^{b}\right)}{\log n}=1-\alpha b, \quad \lim _{n \rightarrow \infty} \frac{\log \mathbb{P}\left(M_{n}^{(\alpha)}>n^{b}\right)}{\log n}=1-\alpha b .
$$

Proof. See [9, Proof of Theorem 6.1].

For a geometric size deviation, we have the following result.

Theorem 5. ([9, Theorem 6.2].) For all $0<\alpha \leq 1, \varepsilon>0$, and $b>1$, we have

$$
\begin{gathered}
\lim _{n \rightarrow \infty} \frac{\log \mathbb{P}\left(X^{(\alpha)}>\varepsilon b^{n / \alpha}\right)}{\log \left(\varepsilon b^{n / \alpha}\right)}=-\alpha, \\
\lim _{n \rightarrow \infty} \frac{\log \mathbb{P}\left(M_{n}^{(\alpha)}>\varepsilon b^{n / \alpha}\right)}{n}=-\log b .
\end{gathered}
$$

Moreover, if $b>1 / \alpha$ then we have

$$
\lim _{n \rightarrow \infty} \frac{\log \mathbb{P}\left(S_{n}^{(\alpha)}>\varepsilon b^{n / \alpha}\right)}{n}=-\log b .
$$

Proof. The proof follows the same arguments as those given in the proof of [9, Theorem 6.2]; however, as that proof contains some trivial errors, we provide a short proof here. Equation (29) follows from (20). By [8, Equation (6.2.1)] we have

$$
\frac{1}{2} n \mathbb{P}\left(X^{(\alpha)}>\varepsilon b^{n / \alpha}\right) \leq \mathbb{P}\left(M_{n}^{(\alpha)}>\varepsilon b^{n / \alpha}\right) \leq n \mathbb{P}\left(X^{(\alpha)}>\varepsilon b^{n / \alpha}\right) .
$$

By (29) we have

$$
\lim _{n \rightarrow \infty} \frac{\log \mathbb{P}\left(X^{(\alpha)}>\varepsilon b^{n / \alpha}\right)}{n}=-\alpha \lim _{n \rightarrow \infty} \frac{\log \left(\varepsilon b^{n / \alpha}\right)}{n}=-\log b,
$$

completing the proof of (30). 
Finally, we check (31). Since $S_{n}^{(\alpha)} \geq M_{n}^{(\alpha)}$, we have $\liminf _{n \rightarrow \infty} n^{-1} \log \mathbb{P}\left(S_{n}^{(\alpha)}>\varepsilon b^{n / \alpha}\right) \geq$ $-\log b$, because of (30). By (19), for $0<1 / b<\alpha \leq 1$, we have $\mathbb{E}\left(\left(X^{(\alpha)}\right)^{1 / b}\right)<\infty$. Therefore, we can apply [10, Lemma 3.2], namely,

$$
\mathbb{P}\left(S_{n}^{(\alpha)}>t^{b}\right) \leq n \mathbb{P}\left(X^{(\alpha)}>\left(\frac{t}{s}\right)^{b}\right)+\mathrm{e}^{s}\left\{\mathbb{E}\left(\left(X^{(\alpha)}\right)^{1 / b}\right)\right\}^{s^{b}}\left(\frac{n}{t}\right)^{s^{b}} \quad \text { for all } s>0 \text { and } t>0 .
$$

Putting $t=\left(\varepsilon b^{n / \alpha}\right)^{1 / b}$ and $s=b^{1 / b}$, we have

$$
\mathbb{P}\left(S_{n}^{(\alpha)}>\varepsilon b^{n / \alpha}\right) \leq(1+o(1)) \sqrt{\frac{2}{\pi}} b^{\alpha-n} n+\frac{\mathrm{e}^{s}}{\varepsilon}\left\{\mathbb{E}\left(\left(X^{(\alpha)}\right)^{1 / b}\right)\right\}^{b} n^{b} b^{-n / \alpha}
$$

for sufficiently large $n$. By the same arguments as those in [16, p. 565] we have

$$
\limsup _{n \rightarrow \infty} n^{-1} \log \mathbb{P}\left(S_{n}^{(\alpha)}>\varepsilon b^{n / \alpha}\right) \leq-\log b .
$$

Hence, we obtain the desired results.

\section{Acknowledgements}

The authors would like to thank the anonymous referee for helpful comments and suggestions. The second author thanks Professors Koichiro Iwata and Shigeo Takenaka for their kind hospitality during his visit to Hiroshima and Okayama, respectively. This research was supported by KAKENHI 21540133 of the Japan Society for the Promotion of Science and the ISM Cooperative Research Program (2012·ISM.CRP-5008).

\section{References}

[1] AdLer, A. (1990). Generalized one-sided laws of the iterated logarithm for random variables barely with or without finite mean. J. Theoret. Prob. 3, 587-597.

[2] Breiman, L. (1992). Probability. SIAM, Philadelphia, PA.

[3] Bingham, N. H., Goldie, C. M. and Teugels, J. L. (1989). Regular Variation. Cambridge University Press.

[4] Durrett, R. (2010). Probability: Theory and Examples, 4th edn. Cambridge University Press.

[5] Feller, W. (1957). An Introduction to Probability Theory and Its Applications, Vol. I, 2nd edn. John Wiley, New York.

[6] Grimmett, G. and Stirzaker, D. (2001). Probability and Random Processes, 3rd edn. Oxford University Press, New York.

[7] Gut, A. (2004). An extension of the Kolmogorov-Feller weak law of large numbers with an application to the St. Petersburg game. J. Theoret. Prob. 17, 769-779.

[8] Gut, A. (2005). Probability: A Graduate Course. Springer, New York.

[9] Gut, A. (2010). Limit theorems for a generalized St. Petersburg game. J. Appl. Prob. 47, 752-760.

[10] Hu, Y. AND Nyrhinen, H. (2004). Large deviations view points for heavy-tailed random walks. J. Theoret. Prob. 17, 761-768.

[11] Maller, R. A. (1978). Relative stability and the strong law of large numbers. Z. Wahrscheinlichkeitsth. 43, $141-148$.

[12] Martin-Löf, A. (1985). A limit theorem which clarifies the 'Petersburg paradox'. J. Appl. Prob. 22, 634-643.

[13] Nolan, J. (2012). Stable Distributions: Models for Heavy Tailed Data. Birkhäuser, Boston, MA.

[14] Norris, J. R. (1997). Markov Chains. Cambridge University Press.

[15] RÉnYI, A. (2007). Foundations of Probability. Dover Publications.

[16] Stoica, G. (2008). Large gains in the St. Petersburg game. C. R. Math. Acad. Sci. Paris 346, 563-566.

[17] Zolotarev, V. M. (1986). One-Dimensional Stable Distributions. American Mathematical Society, Providence, RI. 